Article

\title{
Establishment of an In Vivo Xenograft Mouse Model of a Subcutaneous Submillimeter HT-29 Tumor Formed from a Single Spheroid Transplanted Using Radiation-Crosslinked Gelatin Hydrogel Microwell
}

\author{
Tomoko Tachibana ${ }^{1}$, Tomoko Gowa Oyama ${ }^{2} \mathbb{D}$, Yukie Yoshii ${ }^{1}{ }^{*}$, Fukiko Hihara ${ }^{1}$, Chika Igarashi ${ }^{1}$, \\ Atsushi B Tsuji ${ }^{1}$ (D), Tatsuya Higashi ${ }^{1}$ and Mitsumasa Taguchi ${ }^{2}$ (D) \\ 1 National Institute of Radiological Sciences, National Institutes for Quantum and Radiological Science and \\ Technology (QST), Chiba-shi 263-8555, Japan; tachibana.tomoko@qst.go.jp (T.T.); \\ hihara.fukiko@qst.go.jp (F.H.); igarashi.chika@qst.go.jp (C.I.); tsuji.atsushi@qst.go.jp (A.B.T.); \\ higashi.tatsuya@qst.go.jp (T.H.) \\ 2 Quantum Beam Science Research Directorate, National Institutes for Quantum and Radiological Science and \\ Technology (QST), Takasaki-shi 370-1292, Japan; ohyama.tomoko@qst.go.jp (T.G.O.); \\ taguchi.mitsumasa@qst.go.jp (M.T.) \\ * Correspondence: yoshii.yukie@qst.go.jp
}

check for updates

Citation: Tachibana, T.; Oyama, T.G.; Yoshii, Y.; Hihara, F.; Igarashi, C.; Tsuji, A.B; Higashi, T.; Taguchi, M. Establishment of an In Vivo Xenograft Mouse Model of a Subcutaneous Submillimeter HT-29 Tumor Formed from a Single Spheroid Transplanted Using Radiation-Crosslinked Gelatin Hydrogel Microwell. Appl. Sci. 2021, 11, 7031. https://doi.org/10.3390/ app11157031

Academic Editors: Francisco Arrebola and Qi-Huang Zheng

Received: 16 June 2021

Accepted: 26 July 2021

Published: 30 July 2021

Publisher's Note: MDPI stays neutral with regard to jurisdictional claims in published maps and institutional affiliations.

Copyright: (c) 2021 by the authors. Licensee MDPI, Basel, Switzerland. This article is an open access article distributed under the terms and conditions of the Creative Commons Attribution (CC BY) license (https:// creativecommons.org/licenses/by/ $4.0 /)$.

\begin{abstract}
Colorectal cancer is a frequent cause of death worldwide. The detection and treatment of small nodules are crucial for improving survival of colorectal cancer patients. Submillimeter tumors are useful tools for developing novel methods to approach this issue. However, there are no suitable in vivo models that allow easy monitoring of the growth of these tumors. This study established a xenograft mouse model of subcutaneous submillimeter tumors with human colorectal cancer HT-29 cells. We transplanted a single spheroid formed by HT-29 cells expressing red fluorescent protein (RFP) (HT-29-RFP). Additionally, we adopted our newly developed radiation-crosslinked gelatin hydrogel microwells (rGHMs), which can be used as a culture base to form spheroids and as a transplantation scaffold with biocompatibility and biodegradability. Spheroids approximately $700 \mu \mathrm{m}$ in size were uniformly created in seven days in the respective rGHMs. Every single spheroid was extracted either with or without rGHM and transplanted into the subcutis of severe combined immunodeficiency (SCID) mice $(n=4)$. After 21 days, the spheroids inoculated together with rGHM successfully formed uniform subcutaneous submillimeter tumor xenografts that were observable in vivo in a stereoscopic fluorescence microscope in all transplanted mice. In contrast, spheroids transplanted without rGHM also developed small tumors in all mice but showed higher variability in size than those transplanted with rGHM. During transplantation, the rGHM ensured easy handling and stabilization of the position of a single spheroid. Inoculation of spheroids with rGHM in the nude mice was similarly examined $(n=4)$, showing that only one out of four mice formed tumors. In conclusion, rGHM effectively formed spheroids and created uniformed xenografted submillimeter tumors of HT-29-RFP in SCID mice. Our model could provide a useful platform to develop medicines and methods for detection and treatment of small nodules of colorectal cancer.
\end{abstract}

Keywords: in vivo; radiation-crosslinked gelatin hydrogel; microwell array; spheroid; subcutaneous submillimeter tumor xenograft

\section{Introduction}

Colorectal cancer is the second leading cause of cancer death in the world [1]. For the treatment of this disease, surgery with adjuvant chemotherapy is a standard treatment in clinical practice; however, small nodules that are not easily visible with the naked eye are reportedly a significant poor prognostic factor [2]. Therefore, detection and treatment 
of such small nodules are important for improving the survival of colorectal cancer patients [2]. Small submillimeter tumors are useful tools for developing novel medicines and methods for detecting or treating small cancer nodules [3]. However, the establishment of useful in vivo tumor xenograft models that are applicable to study submillimeter tumors is challenging [4]. In conventional studies, in vivo xenograft mouse models of subcutaneous tumors approximately $5 \mathrm{~mm}$ in size $\left(65 \mathrm{~mm}^{3}\right.$ in volume), made by subcutaneous injection of colorectal cancer cell suspension, have been generally used. Such models serve as powerful tools to investigate treatment efficacy and drug delivery to tumors because it is easy to observe tumor growth and dynamics in vivo using imaging technology $[5,6]$. Previous studies using these models have provided many drugs for treating primary solid tumors $[5,6]$. However, a conventional subcutaneous injection of cancer cell suspension makes it difficult to construct submillimeter tumors because cancer cells cannot localize in very small regions [7]. On the other hand, some in vivo mouse models, such as intraperitoneal disseminated models and metastatic models, have been used to study submillimeter tumors of colorectal cancer [8-10]. These models are constructed by intraperitoneal or intravenous injection of cancer cell suspension and are important for understanding behavior of submillimeter tumors in organs such as, the peritoneal cavity, liver, lung, etc. [8-10]. However, in these models, in vivo observation of the growth of each tumor, especially of submillimeter tumors, is difficult, and tumor growth regulation is challenging.

Thus far, various in vitro 3D culture systems have been reported as practical tools to generate uniform multicellular cancer spheroids [11-15]. Here, we hypothesized that transplantation of a single spheroid cultured using the in vitro 3D culture system would become a core to create a subcutaneous submillimeter tumor xenograft in vivo. For this purpose, we adopted our newly developed radiation-crosslinked gelatin hydrogel microwell (rGHM) arrays [16,17] which can be used as scaffolds for both spheroid culture and transplantation. The rGHM arrays are produced by applying a radiation-molding technique, which forms micropatterned radiation-crosslinked gelatin hydrogels $[16,17]$. In rGHM, gelatin is used as the base material because it is a hydrolyzed form of collagen, the extracellular matrix's main constituent. The radiation-molding technique requires no additives or crosslinkers and, thus, does not alter the biocompatibility and biodegradability of gelatin. Although the sol-gel transition of native gelatin occurs around the mammalian body temperature, the rGHM arrays made of crosslinked gelatin hydrogel do not melt even at $50{ }^{\circ} \mathrm{C}$ and can be stably used for cell culture. Another advantage of rGHM arrays that distinguishes them from other similar technologies is their applicability as a transplantation scaffold. The rGHM arrays allow one to produce single spheroids in the respective rGHMs and transplant them together.

This study aimed to establish an in vivo xenograft mouse model of a subcutaneous submillimeter tumor of human colorectal cancer HT-29 cells expressing red fluorescent protein (RFP) (HT-29-RFP), using subcutaneous transplantation of a single spheroid made of cancer cells together with rGHM as a scaffold.

\section{Materials and Methods}

\subsection{Preparation of rGHM Arrays}

The rGHM arrays (shown in Figure 1) were prepared using a previously reported radiation-molding technique [16,17]. Briefly, gelatin (porcine skin, Type A, G1890; SigmaAldrich, St. Louis, MO, USA) was dissolved in deionized water at $10 \% w / w$ at $50{ }^{\circ} \mathrm{C}$. After pouring the solution into cell culture dishes $(60 \mathrm{~mm}$, Iwaki 1010-060; AGC Techno Glass, Shizuoka, Japan), micropatterned flexible polydimethylsiloxane molds loaded onto a polyethylene-terephthalate film were placed on top of the solution. The samples were stored overnight at $20^{\circ} \mathrm{C}$ to undergo physical gelation. The samples in sealed plastic bags were irradiated using ${ }^{60} \mathrm{Co} \gamma$-ray $\left({ }^{60} \mathrm{Co}\right.$ No. 2 Irradiation Facility of Takasaki Advanced Radiation Research Institute, QST) in the air at $15-20{ }^{\circ} \mathrm{C}$ at $15 \mathrm{~J} / \mathrm{g}(\mathrm{J} / \mathrm{g}=\mathrm{kGy})$. This irradiation process altered the gelatin solution into sterilized rGHM arrays of 20 roundbottomed microwells ( $800 \mu \mathrm{m}$ in diameter and $800 \mu \mathrm{m}$ in depth). 

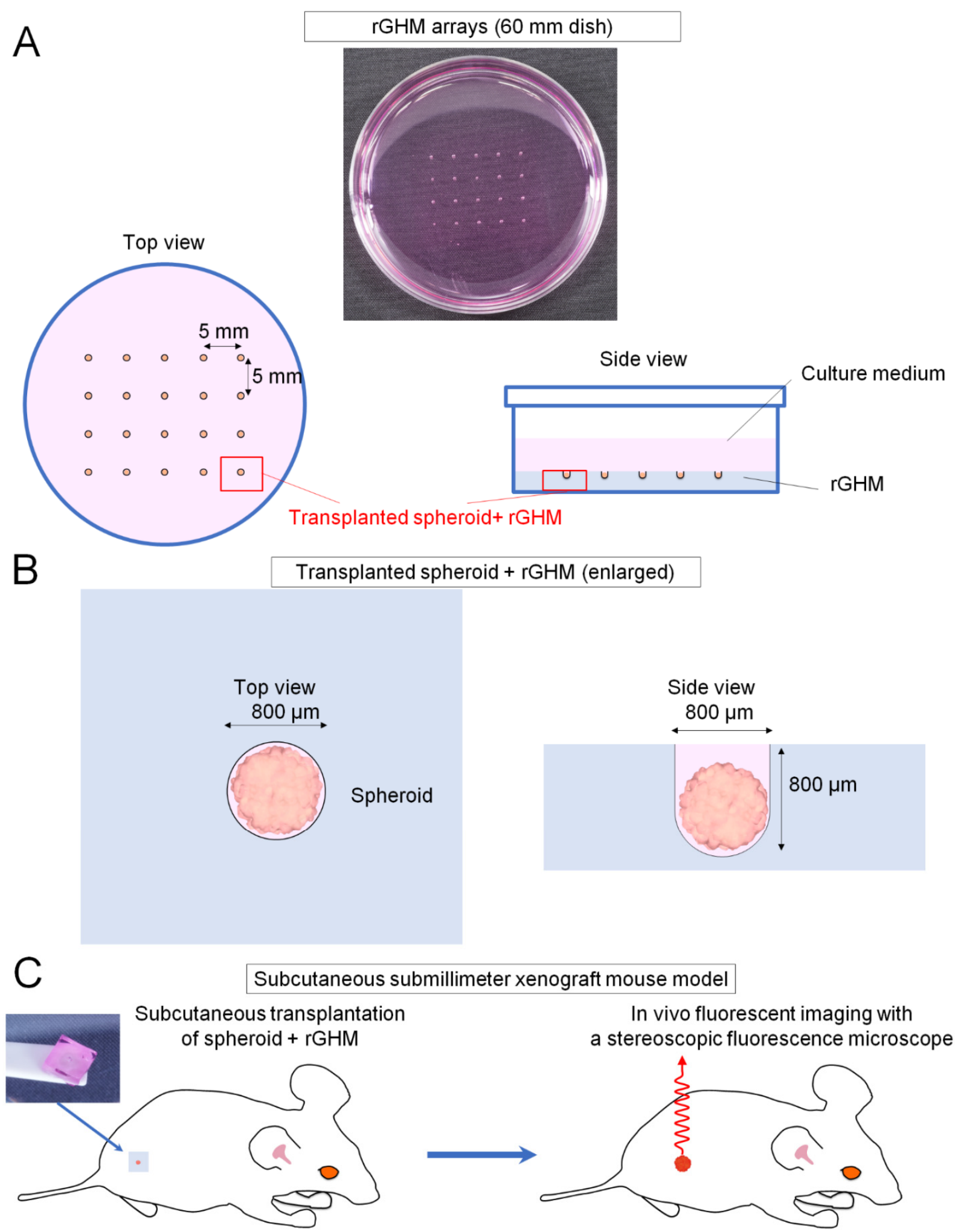

Figure 1. rGHM array and in vivo xenograft mouse model of a subcutaneous submillimeter tumor made by transplantation of a single spheroid together with rGHM. (A) Photograph of rGHM array (upper). The top and side view of rGHM array formed in a $60 \mathrm{~mm}$ dish (lower left and right). Please see the main text for details. Red rectangle indicates the part transplanted into mice. (B) The enlarged top and side view of the part transplanted into mice, which includes a single spheroid cultured in rGHM. (C) Subcutaneous transplantation of a single spheroid with rGHM shown in (B), to make the xenograft mouse model of a subcutaneous submillimeter tumor. The submillimeter xenograft tumor can be observed using fluorescence in vivo imaging in a stereoscopic fluorescence microscope. 


\subsection{Cell Cultivation and Culture of Spheroids}

Human colorectal carcinoma HT-29 cells were obtained from the American Type Culture Collection, Manassas, VA, USA. HT-29 cells were stably transfected with red fluorescent protein (RFP) lentivirus (Lenti-Red, LG502, Biogenova, Rockville, MD, USA) following the manufacturer's protocol. Then, a clone that strongly expressed the RFP was established by limiting dilution and denoted as HT-29-RFP, and these cells were used in this study. The HT-29-RFP cells were cultured in Dulbecco's modified Eagle medium (DMEM) (Wako) supplemented with $10 \%$ fetal bovine serum (culture medium) in a humidified atmosphere with $5 \% \mathrm{CO}_{2}$ at $37^{\circ} \mathrm{C}$. Exponentially growing cells were detached from the plates using trypsin, and the number of viable cells was determined using the Trypan blue dye-exclusion method.

For the pretreatment before seeding the cells, the rGHM arrays were preincubated with $5 \mathrm{~mL}$ of phosphate-buffered saline (PBS) at $50^{\circ} \mathrm{C}$ for $2 \mathrm{~h}$ exchanging the solution twice to remove non-cross-linked components and to allow them to reach an equilibrium phase, and then incubated with $5 \mathrm{~mL}$ DMEM at $37^{\circ} \mathrm{C}$ for 30 min exchanging the medium twice to replace the absorbed PBS with a medium.

After pretreatment, HT-29-RFP cells were seeded in $2.0 \times 10^{6}$ cells in $5 \mathrm{~mL}$ medium per $60 \mathrm{~mm}$ dish equipped with rGHM arrays and cultured for 7 days to obtain spheroids. The obtained spheroids were immediately subjected to transplantation into mice. During the incubation, the establishment of the spheroid morphology was monitored using an inverted microscope (Olympus BX43, Tokyo, Japan) equipped with a camera system (Olympus, DP21, Tokyo, Japan).

\subsection{Xenograft Mouse Model of a Submillimeter Subcutaneous Tumor}

All animal experimental procedures were approved by the Animal Ethics Committee of the National Institutes for Quantum and Radiological Science and Technology (QST, Chiba, Japan) on 2018 (approval no. 13-1022-7) and conducted in accordance with the institutional guidelines.

\subsubsection{Experiment 1}

In this experiment, transplantation of spheroids either with or without rGHM was tested using severe combined immunodeficiency (SCID) (CB17.Cg-PrkdcscidLystbg-J/CrlCrlj) mice. Six-week-old female SCID mice were obtained from the Japan Charles River (Kanagawa, Japan). Mice were acclimatized for at least one week before the experiments and were fed and watered ad libitum. A purified diet (AIN-93M, TestDiet, St. Louis, MO, USA) was used during the experiments to avoid the effect of diet on abdominal autofluorescence detected by in vivo fluorescence microscopic imaging. Transplantation of spheroids either with or without rGHM was tested on the subcutis of the right or left flank in each mouse $(n=4)$. Before transplantation, mice were anesthetized with isoflurane, shaved, and disinfected in the surgery area. For the transplantation of spheroids together with rGHM, an rGHM with a single spheroid located in the center was cut using a surgical scalpel blade to make approximately a $5 \mathrm{~mm}$ square, and subcutaneously transplanted using a spatula onto the mouse's right flank through the incision. For comparison, the transplantation of spheroids without rGHM was also tested; every single spheroid was isolated from the rGHM using aspiration with a micropipette and transplanted onto the mouse's left flank through the incision. The skin incision was closed using sutures.

\subsubsection{Experiment 2}

In the next experiment, transplantation of spheroids together with rGHM was investigated using nude (BALB/cSlc-nu/nu) mice. Six-week-old female nude mice were obtained from Japan SLC (Shizuoka, Japan) and used in this study. Mice were acclimatized for at least one week before the experiments, fed a purified diet (AIN-93M) to reduce the background autofluorescence, and watered ad libitum. Transplantation of spheroids 
together with rGHM was conducted on the subcutis of the right flank of each nude mouse $(n=4)$, similar to that in experiment 1 .

\subsection{In Vivo Observation of Tumor Growth}

After the spheroid transplantation, in vivo observation was performed over time using a stereoscopic fluorescence microscope (MZ16F, Leica, Wetzlar, Germany) equipped with a camera system (DFC310FX, Leica). During the in vivo observation, mice were anesthetized with isoflurane. Bright-field and red fluorescent images were obtained at regions of spheroid transplantation and tumor size was measured using the fluorescent images, since tumors could not be clearly detected in the bright-field images. Tumor volume was calculated using the following equation: tumor volume $=$ length $\times$ width $^{2} \times \pi / 6$. In experiment 1 , mice were sacrificed at day 42 , and tumors were isolated and weighed.

\subsection{Statistical Analysis}

Data were expressed as mean and standard deviation. $p$-values were calculated using a 2-tailed $t$-test for comparison between two groups. Tumor growth curves were analyzed using two-way analysis of variance. $p$-values $<0.05$ were considered statistically significant. Data analyses were conducted using JMP 13.2.0 (SAS Institute, Cary, NA, USA).

\section{Results}

\subsection{Formation of HT-29-RFP Spheroids on the rGHM Array}

In this study, HT-29-RFP cells were seeded at $2.0 \times 10^{6}$ cells in a $5 \mathrm{~mL}$ culture medium per $60 \mathrm{~mm}$ dish with rGHM arrays for seven days. Figure 2 shows representative images of microscopic observation of the morphological changes in a HT-29-RFP spheroid cultured on rGHM array over time. On day 0, cell aggregation was observed at the bottom of the well after the HT-29-RFP cells were seeded on the rGHM arrays (Figure 2, left column). On day 4, growth of the cell aggregates was observed (Figure 2, middle column). On day 7, spheroids of approximately $700 \mu \mathrm{m}$ in diameter were formed (Figure 2, right column). We observed that this system allowed the simultaneous formation of spheroids of uniform size and shape in different wells on day 7 . From the inverted fluorescence microscope observation, the HT-29-RFP cells in the spheroids uniformly emitted high-intensity RFP fluorescent signals.

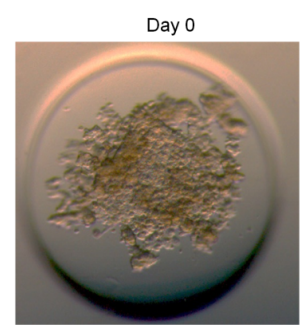

Red Fluorescence

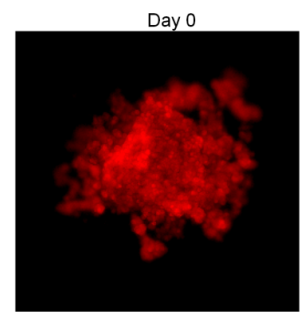

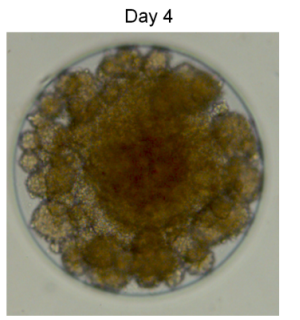

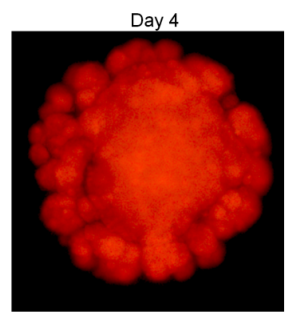

Day 7
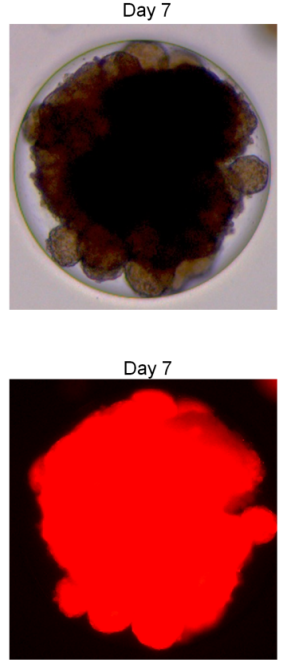

$500 \mu \mathrm{m}$

Figure 2. Observation of the HT-29-RFP spheroids formed in rGHM array. Representative images of spheroid formation by the HT-29-RFP cells in rGHM over time (day 0, day 4, and day 7). Images obtained in an inverted fluorescence microscope are shown. Bright-field and red fluorescence views are shown in the upper and lower parts of the image, respectively. 


\subsection{Observation of an In Vivo Model of a Subcutaneous Submillimeter Xenograft Tumor}

\subsubsection{Experiment 1}

In this experiment, transplantation of spheroids either with or without rGHM in SCID mice was successfully performed $(n=4)$. No body weight loss caused by the surgical procedures was observed in any of the examined mice. For the transplantation of a single spheroid, rGHM was helpful for easy handling and position stabilization of the spheroid, which was more difficult to achieve without rGHMs. We observed that the appearance of the gel under the skin completely disappeared by day 7 after transplantation. Figures 3 and 4 show the in vivo observation of tumor growth after transplantation of HT-29-RFP spheroids either with or without rGHM in SCID mice. The in vivo observation was performed using a stereoscopic fluorescence microscope. From the images acquired at day 0 (just after spheroid transplantation) and day 14, no tumor RFP signals were detected at any spheroid transplantation sites either with or without rGHM (Figure 3). From the images acquired at day 21, RFP signals of small tumors from all spheroid transplantation sites were observed in the examined mice; the tumor major axis was $0.55 \pm 0.20 \mathrm{~mm}$ (coefficient of variation $(\mathrm{CV})=0.37)$ and $1.48 \pm 1.33 \mathrm{~mm}(\mathrm{CV}=0.90)$ and tumor volume was $0.08 \pm 0.06 \mathrm{~mm}^{3}(\mathrm{CV}=0.70)$ and $3.94 \pm 5.80 \mathrm{~mm}^{3}(\mathrm{CV}=1.47)$, for the spheroids transplanted with or without rGHM, respectively; the spheroids transplanted together with rGHM formed subcutaneous submillimeter xenograft tumors at day 21 that showed lower variability in tumor major axis and volume (with a smaller $\mathrm{CV}$ ) than those formed from spheroids transplanted without the rGHM (0.41-fold in tumor major axis and 0.48 -fold in tumor volume). The tumors were actively grown thereafter, as shown in Figures 3 and 4 . There was no significant difference in the tumor growth curve between the groups with or without rGHM (Figure 4). From the measurements of isolated tumor weight at day 42, there was no significant difference in tumor weight, but the $\mathrm{CV}$ in the group with rGHM was 0.47-fold smaller than that in the group without rGHM (Figure 4). These results indicated that the use of rGHM in the transplantation of spheroids helped construct uniform subcutaneous submillimeter xenograft tumors growing uniformly in vivo. 
A
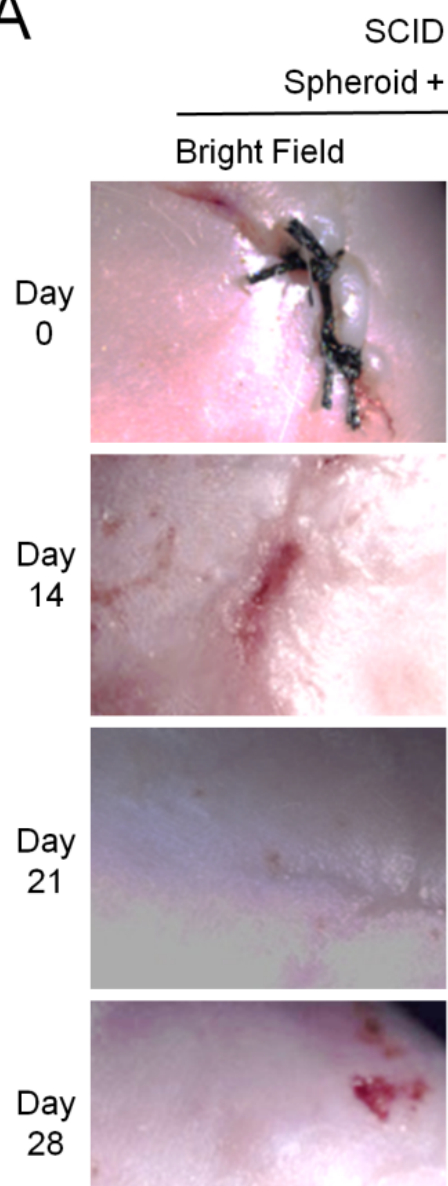

28

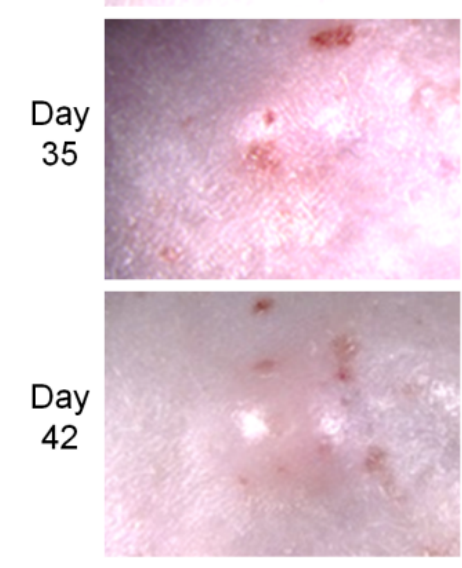

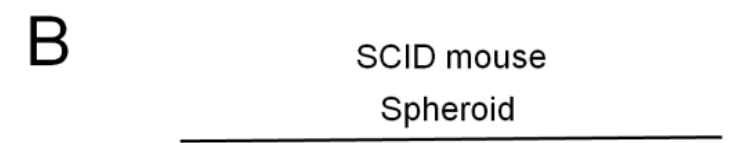
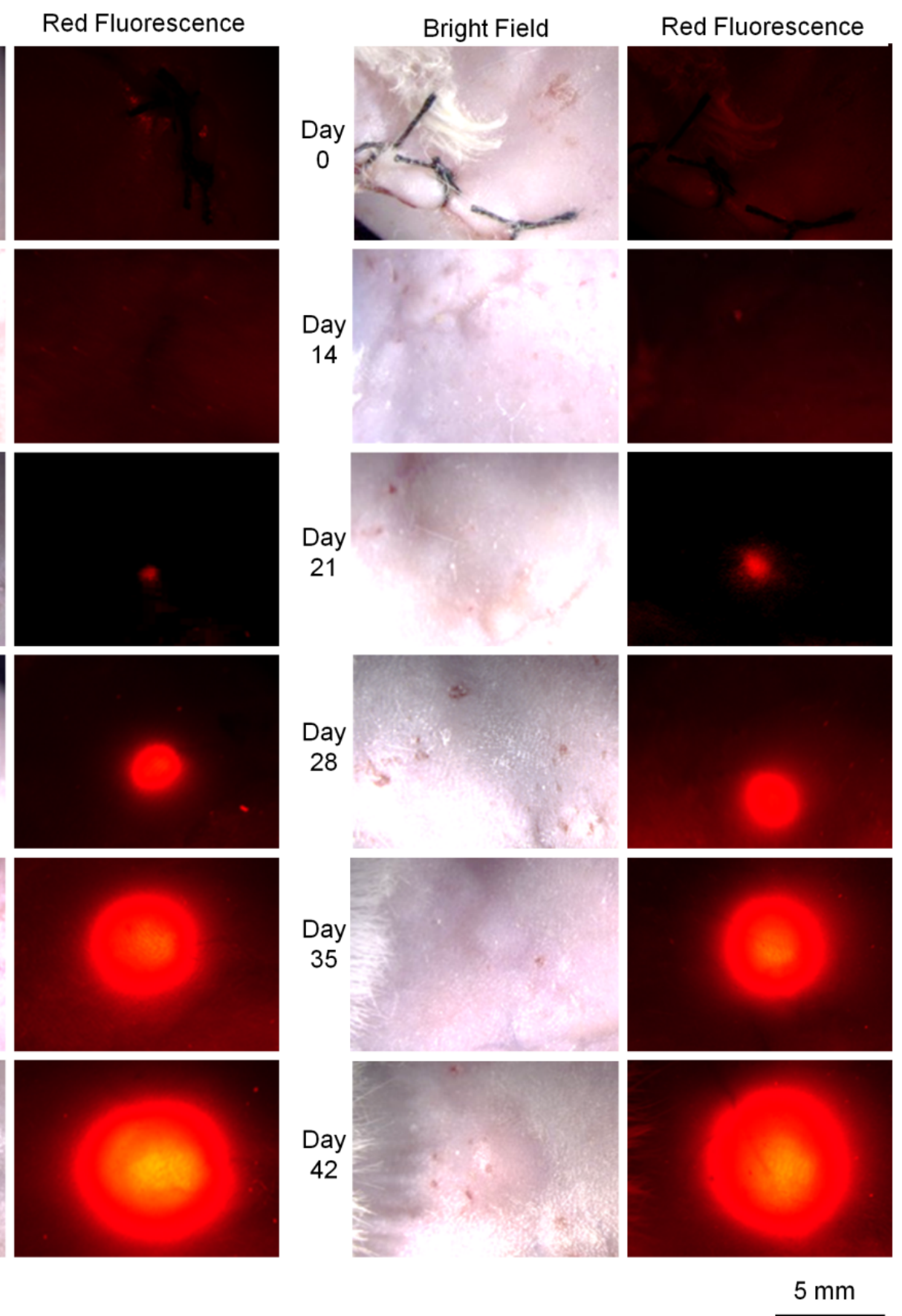

Figure 3. Observation of in vivo xenograft mouse model of a subcutaneous submillimeter HT-29-RFP tumor in SCID mice. (A,B) Representative images of a mouse transplanted with HT-29-RFP spheroids either with or without rGHM, respectively, on day $0,14,21,28,35$, and 42 . Bright-field and red fluorescence views are shown in left and right rows, respectively. 

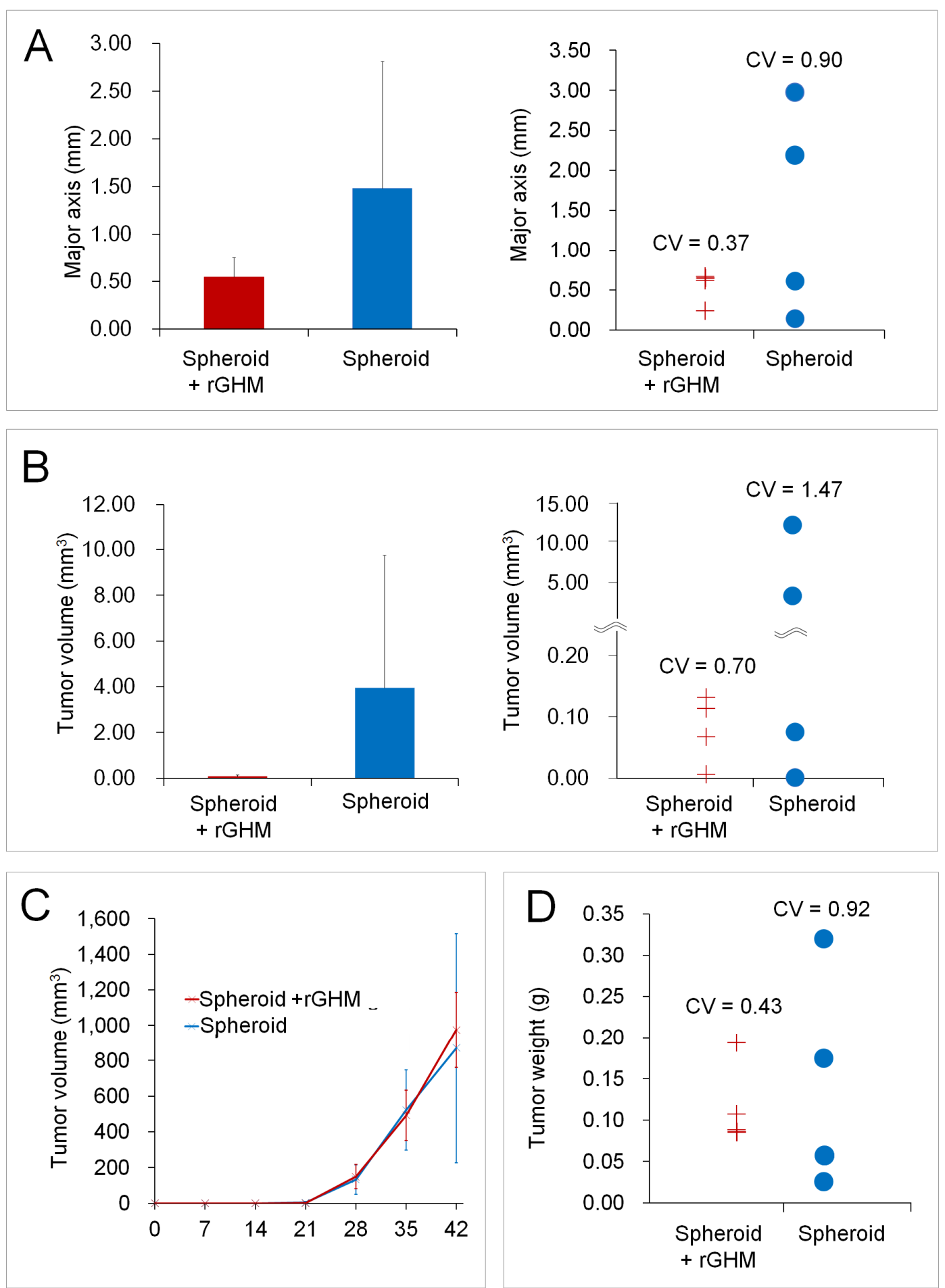

Figure 4. Measurement of tumor parameters in in vivo xenograft mouse model of a subcutaneous submillimeter HT-29-RFP tumor in SCID mice. (A,B) Tumor major axis and volume (left and right) at day 21 with or without rGHM, respectively ( $n=4$ /group). $\mathrm{CV}=$ coefficient of variation. There are no significant differences between the two groups in tumor major axis and volume (a 2-tailed $t$-test), while the values of $\mathrm{CV}$ in the group with rGHM are smaller than those in the group without rGHM (0.41-fold in tumor major axis and 0.48-fold in tumor volume). (C) Changes of tumor volume over time. The $\mathrm{Y}$ axis is scaled to focus on tumor growth at the time point after day 21. There is no significant difference in the tumor growth curve between the two groups (two-way analysis of variance). Weight measurement in isolated tumors at day 42 after transplantation of HT-29-RFP spheroids in SCID mice. (D) Values of the tumor weight in the group with or without rGHM at the transplantation of spheroids. $\mathrm{CV}=$ coefficient of variation. There is no significant difference in tumor weight between the two groups (a 2-tailed $t$-test), while the CV in the group with rGHM is 0.47 -fold smaller than in the group without rGHM. 


\subsubsection{Experiment 2}

Next, the transplantation of spheroids together with rGHM was tested using nude mice $(n=4)$. Figure $5 \mathrm{~A}, \mathrm{~B}$ show representative images of the in vivo observation over time after the transplantation of HT-29-RFP spheroids in nude mice. Similar to that in experiment 1 , the transplantation of spheroids together with rGHM was successfully performed without any obvious adverse signs caused by the surgical procedures in all the examined mice. However, xenograft tumor formation was not observed in three out of four mice ( $75 \%$; Figure $5 \mathrm{~A})$. Only one in four mice (25\%) showed a xenograft tumor formation of $1 \mathrm{~mm}$ in size at day 14, and it continued to grow thereafter (Figure 5B). These results indicated that SCID mice rather than nude mice were appropriate to make an in vivo model of a subcutaneous submillimeter xenograft tumor by transplantation of HT-29-RFP spheroids, since the failure of forming xenografts makes the experiment difficult.

A

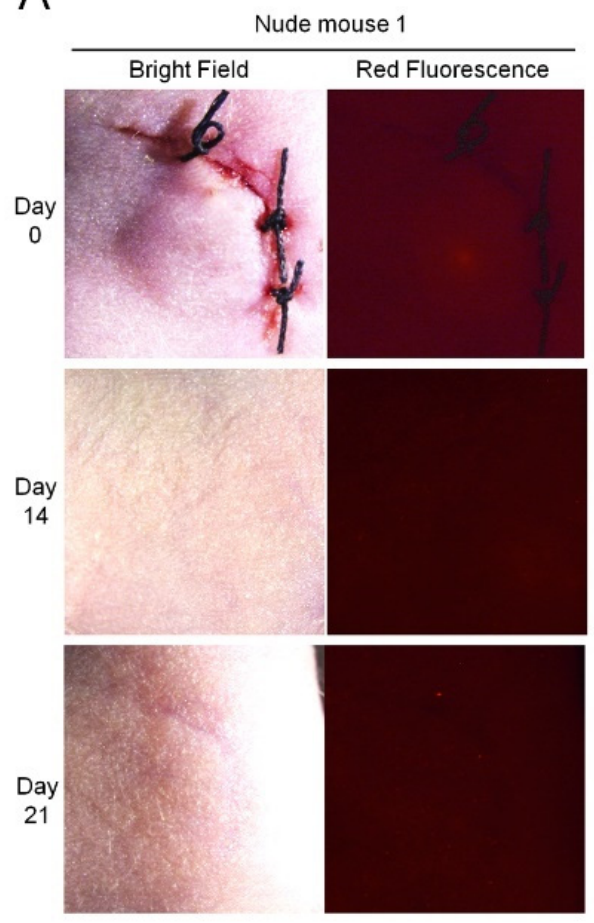

No tumor was observed thereafter.
B

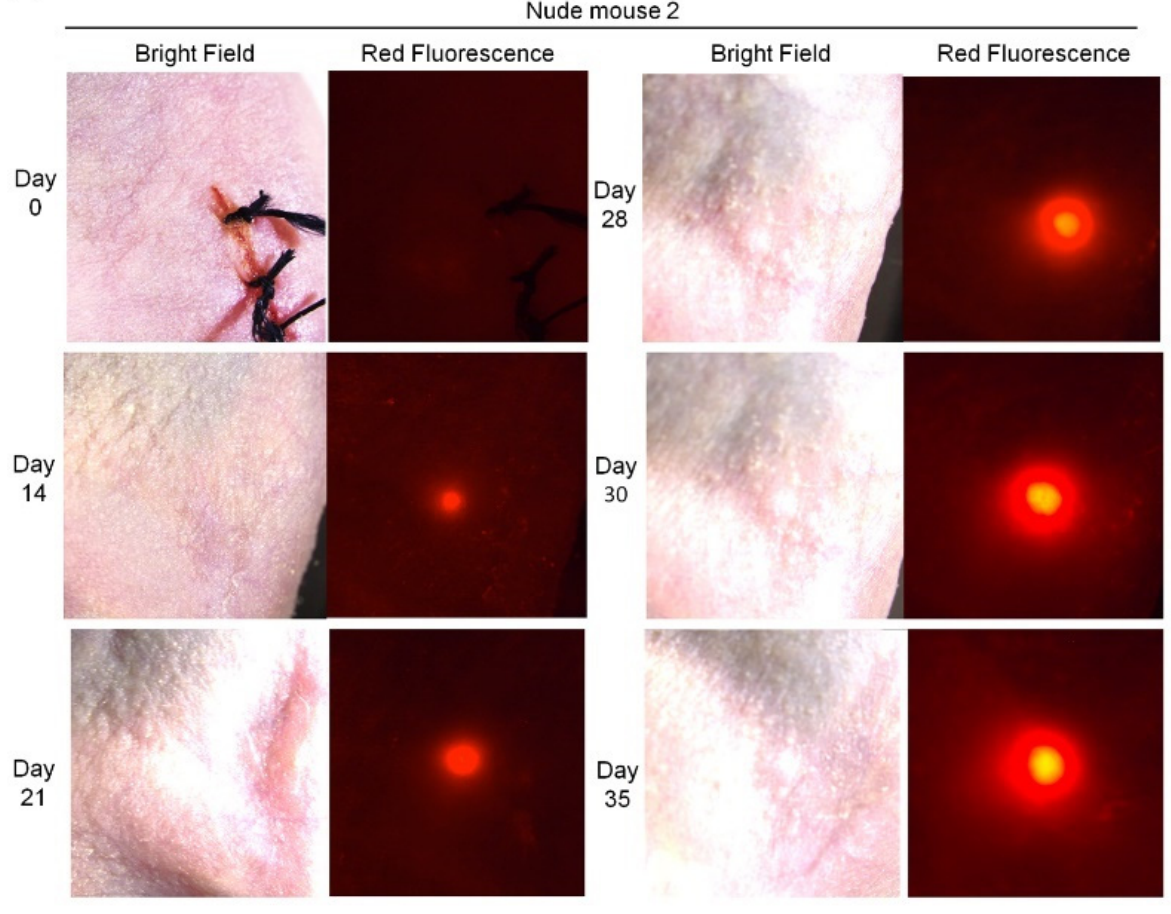

$5 \mathrm{~mm}$

Figure 5. Observation of mice transplanted with HT-29-RFP spheroids cultured on rGHM in nude mice. (A) Representative images of a mouse transplanted with HT-29-RFP spheroids with rGHM, which showed no tumor formation on day 0, 14, and 21, respectively (nude mouse 1). This pattern was observed in three out of four mice (75\%). (B) Representative images of a mouse transplanted with HT-29-RFP spheroids with rGHM, which showed tumor formation on day 0, 14, 21, 28, 30, and 35 , respectively (nude mouse 2). This pattern was observed in one out of four mice (25\%). Bright-field and red fluorescence channels are shown in left and right rows, respectively.

\section{Discussion}

We established a xenograft mouse model of a subcutaneous submillimeter tumor of HT-29-RFP cells by transplanting a single spheroid cultured in biocompatible and biodegradable rGHM in a SCID mouse. By using a stereoscopic fluorescence microscope, submillimeter subcutaneous tumors were easily observed, and tumor growth could be monitored in vivo using this model. Considering that the model established in this study showed uniformity in the tumor shape and timing of the formation of submillimeter xenografts, this model would be a useful tool to develop novel methods and screen drugs for diagnosis and treatment of submillimeter tumors of colorectal cancer. Our subcutaneous submillimeter tumor xenografts were uniformly developed in SCID mice, but not in nude 
mice. The success rate of transplantation of human tumor cells in these mice has been reported to be higher than that in the nude mice due to the mutations present in SCID mice $[18,19]$; therefore, our findings are in agreement with the expectations. This study used a well-characterized human colorectal cancer cell line HT-29 [20], which would be an advantage to use HT-29 in this model.

To date, in vivo subcutaneous xenograft mouse models made by subcutaneous injection of cancer cell suspension have been widely used in many studies on colorectal cancer because of the convenience of such model creation and an easy observation in vivo $[5,6]$. However, the traditional subcutaneous injection of cancer cell suspension is not possible in forming submillimeter tumors in mouse models. In this study, we found that the goal of forming uniform submillimeter tumors in vivo can be successfully achieved by the transplantation of a single spheroid together with rGHM into SCID mice. In this method, the subcutaneous transplantation of a single spheroid was easily performed using rGHM as a scaffold for both spheroid culture and transplantation. We observed that the gel under the skin disappeared a few days after transplantation, suggesting that rGHM underwent enzyme-mediated degradation in vivo, which facilitated nutrition and blood supply from the mouse body to the transplanted spheroids and activated tumor formation. Given these advantages, rGHMs would be useful not only for generating multicellular cancer spheroids but also for developing mouse models with uniform subcutaneous submillimeter tumors. Although rGHM transplantation is more invasive than the subcutaneous injection of cancer cell suspension, the model established in this study has the advantage of producing submillimeter tumors.

As in other conventional models, mouse models constructed by intraperitoneal or intravenous injection of cancer cell suspension have been widely used to study disseminated metastatic small nodules of colorectal cancer [8-10]. These dissemination models are useful tools because they can accurately imitate the disease condition when small tumors are disseminated to organs. However, in vivo identification and observation of each tumor lesion are quite difficult when using these models. The model established in this study achieved in making easily observable and uniformly growing submillimeter tumors by subcutaneous transplantation of a single spheroid cultured in rGHM. From this point of view, this model could compensate for the dissemination model's weakness, and the combined use of these two models would be beneficial for future studies on disseminated metastatic small tumors. For the future perspectives, using patient-derived cells and organoids for this model is promising in order to make more clinically relevant models. Additionally, this model could be applicable to other types of cancers, and future studies would be warranted.

\section{Conclusions}

We established an in vivo xenograft mouse model of a subcutaneous submillimeter tumor with human colorectal cancer HT-29-RFP cells by transplanting a single spheroid with biocompatible and biodegradable rGHM. This model developed easily observable and uniformly growing submillimeter tumors in vivo. This model would provide a useful platform for developing of medicines and methods for the detection and treatment of small nodules of colorectal cancer.

Author Contributions: Y.Y., M.T. and T.G.O. designed the study. T.T., T.G.O., Y.Y., F.H. and C.I. performed the experiments. T.T., Y.Y., M.T., T.G.O., A.B.T. and T.H. wrote the manuscript. All authors also made significant contributions by discussing the results and enhancing the manuscript for intellectual content. All authors have read and agreed to the published version of the manuscript.

Funding: This work was supported by the QST Creative Research Grant from the QST to MT (2020).

Institutional Review Board Statement: All animal experimental procedures were approved by the Animal Ethics Committee of the National Institutes for Quantum and Radiological Science and Technology (QST, Chiba, Japan) on 2018 (approval no. 13-1022-7) and conducted in accordance with the institutional guidelines. 
Informed Consent Statement: Not applicable.

Data Availability Statement: The datasets used during the present study are available from the corresponding author upon reasonable request.

Acknowledgments: This work was supported by the QST Creative Research Grant from the QST to MT. The authors thank Noriko Tawara (QST) and Ryoko Mezaki (QST) for helping with sample preparation, and Ichiro Takahashi (QST) for taking the sample photographs shown in Figure 1.

Conflicts of Interest: The authors declare no conflict of interest.

\section{References}

1. Sung, H.; Ferlay, J.; Siegel, R.L.; Laversanne, M.; Soerjomataram, I.; Jemal, A.; Bray, F. Global cancer statistics 2020: Globocan estimates of incidence and mortality worldwide for 36 cancers in 185 countries. CA Cancer J. Clin. 2021, 71, 209-249. [CrossRef] [PubMed]

2. Sloothaak, D.A.; Sahami, S.; van der Zaag-Loonen, H.J.; van der Zaag, E.S.; Tanis, P.J.; Bemelman, W.A.; Buskens, C.J. The prognostic value of micrometastases and isolated tumour cells in histologically negative lymph nodes of patients with colorectal cancer: A systematic review and meta-analysis. Eur. J. Surg. Oncol. 2014, 40, 263-269. [CrossRef] [PubMed]

3. Georges, L.M.C.; De Wever, O.; Galván, J.A.; Dawson, H.; Lugli, A.; Demetter, P.; Zlobec, I. Cell line derived xenograft mouse models are a suitable in vivo model for studying tumor budding in colorectal cancer. Front. Med. 2019, 6, 139. [CrossRef] [PubMed]

4. Nakagawa, S.; Matsuoka, Y.; Ichihara, H.; Yoshida, H.; Yoshida, K.; Ueoka, R. Therapeutic effects of autologous lymphocytes activated with trastuzumab for xenograft mouse models of human breast cancer. Biol. Pharm. Bull. 2013, 36, 861-865. [CrossRef] [PubMed]

5. Matsumoto, H.I.; Okumura, M.; Matsumoto, Y. Therapeutic effects of hybrid liposomes against xenograft mouse model of colorectal cancer in vivo due to long-term accumulation. Anticancer Res. 2016, 36, 5875-5882.

6. Yi, B.R.; Park, M.A.; Lee, H.R.; Kang, N.H.; Choi, K.J.; Kim, S.U.; Choi, K.C. Suppression of the growth of human colorectal cancer cells by therapeutic stem cells expressing cytosine deaminase and interferon- $\beta$ via their tumor-tropic effect in cellular and xenograft mouse models. Mol. Oncol. 2013, 7, 543-554. [CrossRef] [PubMed]

7. Kingston, B.R.; Syed, A.M.; Ngai, J.; Sindhwani, S.; Chan, W.C.W. Assessing micrometastases as a target for nanoparticles using 3d microscopy and machine learning. Proc. Natl. Acad. Sci. USA 2019, 116, 14937-14946. [CrossRef] [PubMed]

8. Taibi, A.; Albouys, J.; Jacques, J.; Perrin, M.-L.; Yardin, C.; Fontanier, S.D.; Bardet, S.M. Comparison of implantation sites for the development of peritoneal metastasis in a colorectal cancer mouse model using non-invasive bioluminescence imaging. PLoS ONE 2021, 14, e0220360. [CrossRef]

9. Gremonprez, F.; Willaert, W.; Ceelen, W. Animal models of colorectal peritoneal metastasis. Pleura Peritoneum 2016, 1, 23-43. [CrossRef] [PubMed]

10. Sullivan, J.; Medina, J.C.; Collins, T.L.; Schmid-Alliana, A.; Schmid-Antomarchi, H.; Cambien, B.; Karimdjee, B.F.; Richard-Fiardo, P.; Bziouech, H.; Barthel, R.; et al. Organ-specific inhibition of metastatic colon carcinoma by cxcr3 antagonism. Br. J. Cancer 2009, 100, 1755-1764.

11. Yoshii, Y.; Furukawa, T.; Aoyama, H.; Adachi, N.; Zhang, M.R.; Wakizaka, H.; Fujibayashi, Y.; Saga, T. Regorafenib as a potential adjuvant chemotherapy agent in disseminated small colon cancer: Drug selection outcome of a novel screening system using nanoimprinting 3-dimensional culture with hct116-rfp cells. Int. J. Oncol. 2016, 48, 1477-1484. [CrossRef] [PubMed]

12. Rijal, G.; Li, W. A versatile 3d tissue matrix scaffold system for tumor modeling and drug screening. Sci. Adv. 2017, 3, e1700764. [CrossRef] [PubMed]

13. Amaral, R.L.F.; Miranda, M.; Marcato, P.D.; Swiech, K. Comparative analysis of $3 \mathrm{~d}$ bladder tumor spheroids obtained by forced floating and hanging drop methods for drug screening. Front. Physiol. 2017, 8, 605. [CrossRef] [PubMed]

14. Lee, J.M.; Park, D.Y.; Yang, L.; Kim, E.J.; Ahrberg, C.D.; Lee, K.B.; Chung, B.G. Generation of uniform-sized multicellular tumor spheroids using hydrogel microwells for advanced drug screening. Sci. Rep. 2018, 8, 17145. [CrossRef] [PubMed]

15. Li, Y.; Kumacheva, E. Hydrogel microenvironments for cancer spheroid growth and drug screening. Sci. Adv. 2018, 4, eaas8998. [CrossRef] [PubMed]

16. Oyama, T.G.; Kimura, A.; Nagasawa, N.; Oyama, K.; Taguchi, M. Development of advanced biodevices using quantum beam microfabrication technology. Quantum Beam Sci. 2020, 4, 14. [CrossRef]

17. Oyama, T.G.; Oyama, K.; Kimura, A.; Yoshida, F.; Ishida, R.; Yamazaki, M.; Miyoshi, H.; Taguchi, M. Collagen hydrogels with controllable combined cues of elasticity and topography to regulate cellular processes. Biomed. Mater. 2021, 16, 045037. [CrossRef] [PubMed]

18. Thomsen, M.; Galvani, S.; Canivet, C.; Kamar, N.; Bohler, T. Reconstitution of immunodeficient scid/beige mice with human cells: Applications in preclinical studies. Toxicology 2008, 246, 18-23. [CrossRef] [PubMed]

19. Okada, S.; Vaeteewoottacharn, K.; Kariya, R. Application of highly immunocompromised mice for the establishment of patientderived xenograft (pdx) models. Cells 2019, 8, 889. [CrossRef] [PubMed]

20. Ahmed, D.; Eide, P.; Eilertsen, I.; Danielsen, S.; Eknaes, M.; Hektoen, M.; Lind, G.; Lothe, R. Epigenetic and genetic features of 24 colon cancer cell lines. Oncogenesis 2013, 2, e71. [CrossRef] [PubMed] 